\title{
Treatment of uncomplicated gonorrhoea with spectinomycin hydrochloride (Trobicin)
}

\author{
I. A. PORTER AND H. W. RUTHERFORD \\ Regional Laboratory, City Hospital, Aberdeen and the VD Department, Aberdeen Royal Infirmary
}

SUMMARY 110 patients with uncomplicated gonococcal urethritis were treated with $2 \mathrm{~g}$ spectinomycin (Trobicin) intramuscularly. Seventy-five patients were available for follow-up and there was only one treatment failure, giving a cure rate of $98.6 \%$. Of the 99 gonococcal isolates, 59 were sensitive to $5 \mu \mathrm{g} / \mathrm{ml}$ of spectinomycin or less and 40 were sensitive to $10 \mu \mathrm{g} / \mathrm{ml}$. In a small sample of patients $2 \mathrm{~g}$ spectinomycin intramuscularly produced a serum concentration averaging $100 \mu \mathrm{g} / \mathrm{ml}$ after one hour.

\section{Introduction}

In 1960 actinospectacin, a broad spectrum antibiotic produced from Streptomyces spectabilis became available for clinical investigation and $2 \mathrm{~g}$ actinospectacin sulphate intramuscularly was found to give cure rates of $87 \%$ to $95 \%$ (Laird and Taylor, 1962; Willcox, 1963) in uncomplicated gonorrhoea.

Spectinomycin hydrochloride (Trobicin) became available in the USA in 1969. It provides a more concentrated suspension and is more soluble than the sulphate salt. Favourable results have been reported with this drug in the treatment of gonorrhoea. Reyn et al. (1973) reported a cure rate of $99 \%$ in Denmark, Stratigos et al. (1973) a rate of $98.5 \%$ in Greece, Kousa et al. (1974) a rate of $93 \%$ in Finland, and Willcox (1974) reported a cure rate of $91.6 \%$ in Britain.

The parenteral treatment of gonorrhoea with penicillin is still widely used as penicillin is relatively non-toxic, comparatively cheap, and usually effective when given in suitable dosage. The increased incidence of gonorrhoea has however been accompanied by numbers of relatively resistant strains of the gonococcus to penicillin (British Medical Journal, 1972). Allergic reactions to this drug also constitute a problem which precludes its use in certain individuals (Idsoe et al., 1968). Consequently it is desirable that other drugs should be evaluated in the treatment of gonorrhoea and the purpose of this study is to investigate the efficacy of spectino-

Address for reprints: Dr H. W. Rutherford, Special Clinic, Royal Infirmary, Woolmanhill, Aberdeen

Received for publication 10 December 1975 mycin hydrochloride in the treatment of uncomplicated gonorrhoea.

\section{Patients and methods}

The study was carried out on 110 men presenting with acute gonococcal urethritis. The diagnosis of gonorrhoea was made by finding Gram-negative intracellular diplococci in stained smears of urethral discharge, confirmed by culture and sugar fermentation tests. Each patient was given $2 \mathrm{~g}$ spectinomycin hydrochloride in a single intramuscular injection. Ten patients had blood withdrawn one hour after the injection of spectinomycin for estimation of spectinomycin serum levels.

Patients were instructed to avoid sexual intercourse and alcohol and asked to report again in $\mathbf{4 8}$ hours and in seven days. Patients were told to hold their urine for at least three hours before attending for follow-up examination, but not all patients complied. All however were able to produce adequate samples of urine, although some were detained for this and others returned after about an hour. Clinical examination and the two-glass urine test was carried out at all subsequent visits and if the patient had any residual discharge a further Gram-stained smear and specimen for culture were taken. Subsequent follow-up examinations were arranged for the end of the first, second, and third months.

\section{Bacteriological studies}

Urethral discharge from each patient was collected on a charcoal swab and transported to the laboratory 
in Stuart's transport medium. The swabs were investigated within 24 hours of collection and usually within 18 hours.

The swabs were plated on to chocolate agar, and on to chocolate agar containing polymixin $\mathbf{B}$ $100 \mathrm{units} / \mathrm{ml}$ and vancomycin $10 \mu \mathrm{g} / \mathrm{ml}$. The inoculated plates were incubated for four days at a temperature of $37^{\circ} \mathrm{C}$ in an atmosphere of $10 \%$ carbon dioxide in air. Daily inspection of the plates was carried out and any colonies looking like Neisseria gonorrhoeae were examined by Gram staining and the oxidase reaction. Confirmation of $N$. gonorrhoeae was based on the results of sugar fermentation tests.

\section{SPECTINOMYCIN SENSITIVITY TESTS}

Strains of $N$. gonorrhoeae isolated from patients in this trial were tested for their sensitivity to spectinomycin by the method previously described (Porter and Wood, 1974). The minimum inhibitory concentration (MIC) of spectinomycin was taken as the lowest concentration which inhibited the growth of the isolate of $N$. gonorrhoeae under test.

SERUM LEVELS OF SPECTINOMYCIN

Estimation of serum levels from 10 patients one hour after treatment with $2 \mathrm{~g}$ spectinomycin was carried out according to the method devised by Porter and Wood (to be published).

\section{Results of treatment}

Eleven of the original 110 male cases had negative culture results and were excluded from the study. Twelve patients defaulted and were not seen again after treatment and nine patients attended on only two occasions-at 48 hours and after one week; these were also excluded. Three patients became reinfected after re-exposure and were also excluded. This left a total of 75 patients for study. One of these patients after temporary improvement following treatment developed a recurrence of urethritis within a week, confirmed by smear and culture to be gonococcal. He denied fresh exposure to infection and was regarded as a treatment failure. The MIC of spectinomycin to the strain of the gonococcus isolated from this patient was $10 \mu \mathrm{g} / \mathrm{ml}$. The remaining 74 patients completed at least two months' surveillance after treatment with spectinomycin. Thirteen $(17.5 \%)$ of these patients developed post-gonococcal urethritis which may be defined as urethritis persisting or recurring after the elimination of the gonococcus by treatment, confirmed by Gramstained smear and culture; these 13 patients responded to treatment with tetracycline.

None of the remaining 61 patients had any further symptoms after treatment with spectinomycin. The urethral discharge in all cases had stopped within 48 hours and their urine was clear within a week. Only one patient complained of pain due to the injection of spectinomycin and there were no side-effects to the drug.

\section{MINIMUM INHIBITORY CONCENTRATIONS}

Swabs were received from 110 patients with urethral discharge and $N$. gonorrhoeae were isolated from 99 of them; no growth was obtained from 11 swabs. Of the 99 strains available for testing 59 were sensitive to $5 \mu \mathrm{g}$ or less of spectinomycin dihydrochloride, the remaining 40 strains being sensitive to less than $10 \mu \mathrm{g}$ but more than $5 \mu \mathrm{g}$ of this antibiotic.

SERUM LEVELS OF SPECTINOMYCIN

Serum was obtained from 10 patients after treatment with spectinomycin. In one patient the level of spectinomycin in the serum was found to be 112 $\mu \mathrm{g} / \mathrm{ml}$, in nine patients it was $87 \mu \mathrm{g} / \mathrm{ml}$. These blood levels of spectinomycin were well above the MIC of this antibiotic for the strains of $N$. gonorrhoeae isolated from these patients.

\section{Discussion}

Male patients with gonorrhoea were chosen for this study of spectinomycin treatment because it is easier to assess cure in men than in women. Of the 75 cases followed-up there was only one treatment failure, a cure rate of $98.6 \%$ which is almost identical with the $98.5 \%$ rate obtained by Stratigos et al. (1973) in Greece. Willcox (1974) in London, reported a cure rate of $91.6 \%$ but half the failures occurred in Negroes in whom early reinfection may be expected; if this group are excluded the cure rate would be $96.3 \%$. The injection of spectinomycin appeared to cause less discomfort to patients than a similar dose of kanamycin and there were no side-effects.

The MICs of spectinomycin of most of the 99 gonococcal strains were sensitive to $5 \mu \mathrm{g} / \mathrm{ml}$ of spectinomycin or less and the remainder were sensitive to $10 \mu \mathrm{g} / \mathrm{ml}$. The serum concentration from a small sample of patients showed a concentration averaging $100 \mu \mathrm{g} / \mathrm{ml}$ after one hour.

With the high incidence of non-specific urethritis at the present time (Department of Health and Social Security, 1976), post-gonococcal urethritis is a relatively common sequel to successful treatment of gonorrhoea in men. In 1973 there were 68139 reported cases of non-specific urethritis in men, nearly 6000 more than in 1972 and exceeding reported cases of gonorrhoea in men by 30306 in 1973 
(Department of Health and Social Security, 1975.) The number of patients with post-gonococcal urethritis after treatment with spectinomycin was 13 $(17 \cdot 5 \%)$ out of a total of 75 patients followed-up.

We wish to thank Upjohn Ltd for arranging for supplies of Trobicin to be made available for the clinical trial.

\section{References}

British Medical Journal (1972). Editorial: Treatment of gonorrhoea. British Medical Journal, 2, 421-422.

Department of Health and Social Security (1975). Extract from Annual Report of the Chief Medical Officer for 1973. British Journal of Venereal Diseases, 51, 63-68.

Department of Health and Social Security (1976). Extract from Annual Report of the Chief Medical Officer for 1974. British Journal of Venereal Diseases, 52, 351-354.
Idsoe, O., Guthe, T., Willcox, R. R., and De Week, A. L. (1968) Nature and extent of penicillin side reaction, with particular reference to fatalities from anaphylactic shock. Bulletin of the World Health Organization, 38, 159-188.

Kousa, M., Lassus, A., Järveläinen, R., and Renkonen, O.-V. (1974). Spectinomycin hydrochloride in the treatment of uncomplicated gonorrhoea in males and females. British Journal of Venereal Diseases, 50, 291-293.

Laird, S. M., and Taylor, G. (1962). Treatment of gonorrhoea with actinospectacin. British Journal of Venereal Diseases, 38, 60-63.

Porter, I. A., and Wood, W. J. (1974). Spectinomycin minimum inhibitory concentrations for Neisseria gonorrhoeae. British Journal of Venereal Diseases, 50, 289-290.

Reyn, A., Schmidt, M., Trier, M., and Bentzon, M. W. (1973). Spectinomycin hydrochloride (Trobicin) in the treatment of gonorrhoea. British Journal of Venereal Diseases, 49, 54-59.

Stratigos, J. D., Marsellou-Kint, O., Kassimatis, V., and Daikos, G. K. (1973). Treatment of gonorrhoea with spectinomycin hydrochloride. British Journal of Venereal Diseases, 49, 60-61.

Willcox, R. R. (1963). Actinomycin (Trobicin) in the treatment of gonorrhoea. Acta dermato-venereologica, 45, 399-404.

Willcox, R. R. (1974). Spectinomycin in the treatment of gonorrhoea in males. British Journal of Venereal Diseases, 50, 294-297. 